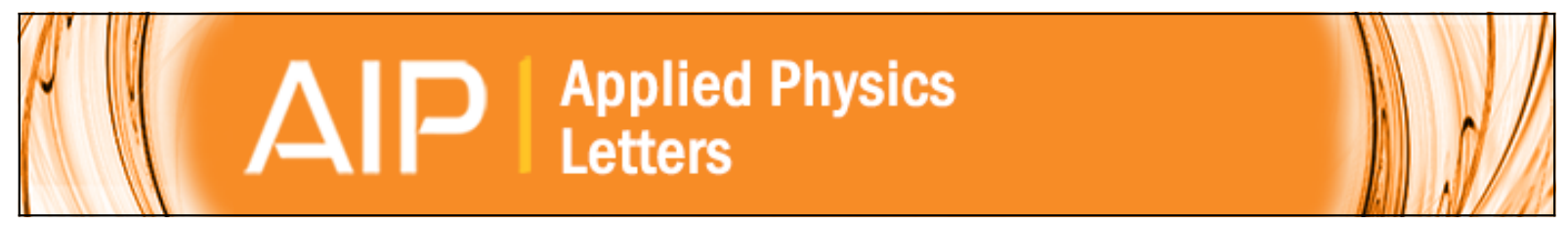

Grain size effect of phase coexistence around morphotropic phase boundary in ferroelectric polycrystalline ceramics

Wei-Feng Rao and Yu U. Wang

Citation: Applied Physics Letters 92, 102905 (2008); doi: 10.1063/1.2896612

View online: http://dx.doi.org/10.1063/1.2896612

View Table of Contents: http://scitation.aip.org/content/aip/journal/apl/92/10?ver=pdfcov

Published by the AIP Publishing 


\title{
Grain size effect of phase coexistence around morphotropic phase boundary in ferroelectric polycrystalline ceramics
}

\author{
Wei-Feng Rao and Yu U. Wang ${ }^{\text {a) }}$ \\ Department of Materials Science and Engineering, Virginia Tech, Blacksburg, Virginia 24061, USA
}

(Received 15 January 2008; accepted 21 February 2008; published online 12 March 2008)

\begin{abstract}
Computer modeling and simulation reveals a grain size- and composition-dependent behavior of phase coexistence around the morphotropic phase boundaries in polycrystals of ferroelectric solid solutions. It shows that the width of phase coexistence composition range increases with decreasing grain sizes; phase-coexisting domain microstructures effectively reduce elastostatic, electrostatic, and domain wall energies; and grain boundaries impose internal mechanical and electric boundary conditions, which affect the phase-coexisting domain microstructures in the grains and give rise to the grain size effect of phase coexistence. (C) 2008 American Institute of Physics.
\end{abstract}

[DOI: $10.1063 / 1.2896612]$

Phase coexistence is a phenomenon commonly observed around the morphotropic phase boundaries (MPBs) in ferroelectric solid solutions, e.g., $\mathrm{Pb}\left(\mathrm{Zr}_{1-x} \mathrm{Ti}_{x}\right) \mathrm{O}_{3} \quad(\mathrm{PZT}){ }^{1}$ $\mathrm{Pb}\left[\left(\mathrm{Zn}_{1 / 3} \mathrm{Nb}_{2 / 3}\right)_{1-x} \mathrm{Ti}_{x}\right] \mathrm{O}_{3},{ }^{2}$ and $\mathrm{Pb}\left[\left(\mathrm{Mg}_{1 / 3} \mathrm{Nb}_{2 / 3}\right)_{1-x} \mathrm{Ti}_{x}\right] \mathrm{O}_{3}{ }^{3}$ The MPB is a nearly vertical (i.e., almost temperature independent) phase boundary in the temperature-composition phase diagram, separating rhombohedral and tetragonal ferroelectric phases at low and high Ti contents, respectively. Because the best piezoelectric properties of these materials are obtained at compositions near their MPBs, ${ }^{1-4}$ the origin of MPB phase coexistence and its effect on the increased piezoelectric response is a topic of both scientific curiosity and technological importance and has been investigated from different perspectives. ${ }^{5-12}$ Recently, intermediate MPB phases were reported based on high-resolution $\mathrm{X}$-ray and neutron diffraction observations, ${ }^{13}$ which renew the interest in the MPB phases. More recently, theoretical ${ }^{14,15}$ and experimental $^{16,17}$ investigations reveal nanodomain aspects of the MPB structures and coherent scattering and interference effects in nanodomain diffractions, underscoring the importance of domain microstructures and mechanisms in the vicinity of MPBs.

Our recent computer modeling and simulation studies of polar domain microstructures in single crystals of MPBbased ferroelectric solid solutions showed that electrostatic and elastostatic interactions play important roles in the MPB phase coexistence phenomenon through the formation of coupled ferroelectric and ferroelastic domain microstructures which are produced by either displacive phase transformation (diffusionless) ${ }^{11}$ or coherent phase decomposition (diffusional). ${ }^{12}$ The phase-coexisting domain microstructures effectively reduces the total system free energy, including bulk free energy, domain wall energy, and long-range electrostatic and elastostatic energies. Similar phase coexistence phenomenon is also reported in the computer simulation of epitaxially strained ferroelectric thin films. ${ }^{18}$ In this work, we study ferroelectric polycrystalline ceramics. The purpose of this letter is to report grain size-dependent behavior and underlying mechanism of phase coexistence in polycrystals, where the width of phase coexistence composition range in${ }^{\text {a) }}$ Author to whom correspondence should be addressed. Electronic mail:
yuwang @mse.vt.edu. creases with decreasing grain sizes. In particular, we investigate the effects of internal mechanical and electric boundary conditions imposed by grain boundaries, which affect the phase-coexisting domain microstructures in the grains. Since diffusion kinetics in the complex oxide solid solutions is slow at room temperature and diffusional process plays only a minor role, we focus on the displacive (diffusionless) evolutions of spontaneous polarization and domain microstructures without local composition change.

The state of a ferroelectric polycrystal is described by a polarization field $\mathbf{P}(\mathbf{r})$. The total system free energy is ${ }^{19}$

$$
\begin{aligned}
F= & \int d^{3} r\left[f\left(R_{i j} P_{j}\right)+\frac{\beta}{2} \frac{\partial P_{i}}{\partial r_{j}} \frac{\partial P_{i}}{\partial r_{j}}\right] \\
& +\frac{1}{2} \int \frac{d^{3} k}{(2 \pi)^{3}}\left[\frac{n_{i} n_{j}}{\varepsilon_{0}} \widetilde{P}_{i} \widetilde{P}_{j}^{*}+K_{i j k l} \widetilde{\varepsilon}_{i j}^{0} \widetilde{\varepsilon}_{k l}^{0 *}\right],
\end{aligned}
$$

which is the same as for a ferroelectric single crystal ${ }^{11}$ except that $\mathbf{P}(\mathbf{r})$ here is defined in a global coordinate system attached to the polycrystalline sample instead of in a local coordinate system aligned with $\langle 100\rangle$ lattice axes of single crystal. $R_{i j}(\mathbf{r})$ is a grain rotation matrix field that describes the geometry (size, shape, and location) and crystallographic orientation of individual grains in the polycrystal, as in the phase field models of martensitic, ${ }^{20}$ ferromagnetic, ${ }^{21}$ and ferroelectric $^{22}$ polycrystals. The operation $R_{i j} P_{j}$ in the Landau-Ginzburg-Devonshire ${ }^{23}$ (LGD) function $f$ transforms $\mathbf{P}(\mathbf{r})$ from global sample system to local crystallographic system in each grain. The gradient, electrostatic, and elastostatic (within isotropic elasticity) energy terms in Eq. (1) as well as the kinetic (time-dependent Ginzburg-Landau) equation are essentially the same as in the model of ferroelectric single crystals. ${ }^{11}$ In this work, we use the material parameters experimentally determined for PZT. ${ }^{24} \mathrm{PZT}$ is the current material of choice for a wide variety of highperformance electromechanical devices. Since PZT is intractable for single crystal growth, polycrystalline ceramics are the material form for practical applications, where grain size effect of phase coexistence is a particularly important issue.

The computer simulations reveal a grain size effect of phase coexistence around the MPB in ferroelectric polycrystals, as shown in Figs. 1 and 2. Two-dimensional polycrystalline structure of 16 grains is generated by using Voronoi 


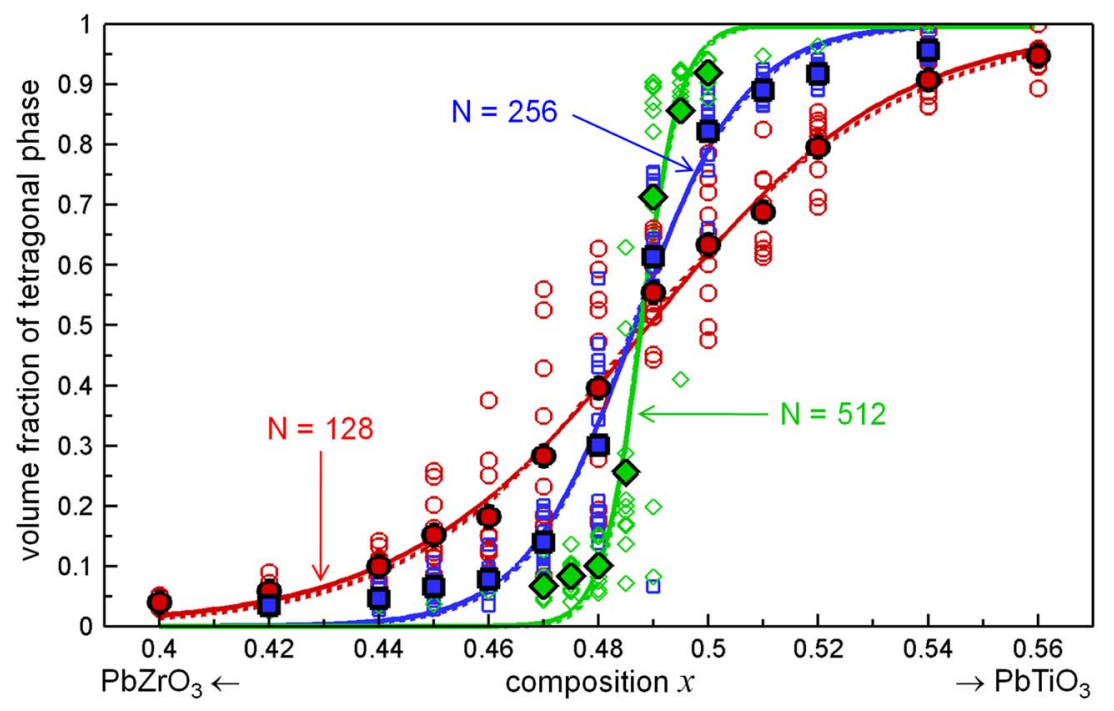

FIG. 1. (Color online) Computer simulation of grain size-dependent phase coexistence phenomenon around MPB in ferroelectric polycrystals. The volume fraction of tetragonal phase is plotted vs the composition $x$ for various grain sizes, as indicated by simulation size $N$. The open symbols represent individual simulation results from random initial conditions. Each of the filled symbols represents the average of ten simulation results for same specific composition and grain size. The solid and dashed lines are the fitting curves by using symmetric and asymmetric functions, respectively.

tessellation $^{20,21}$ with random grain orientations. In order to separate grain size effect from other effects, the same polycrystalline grain structure (i.e., grain shape, location, and orientation) is used in all simulations. Different grain sizes are
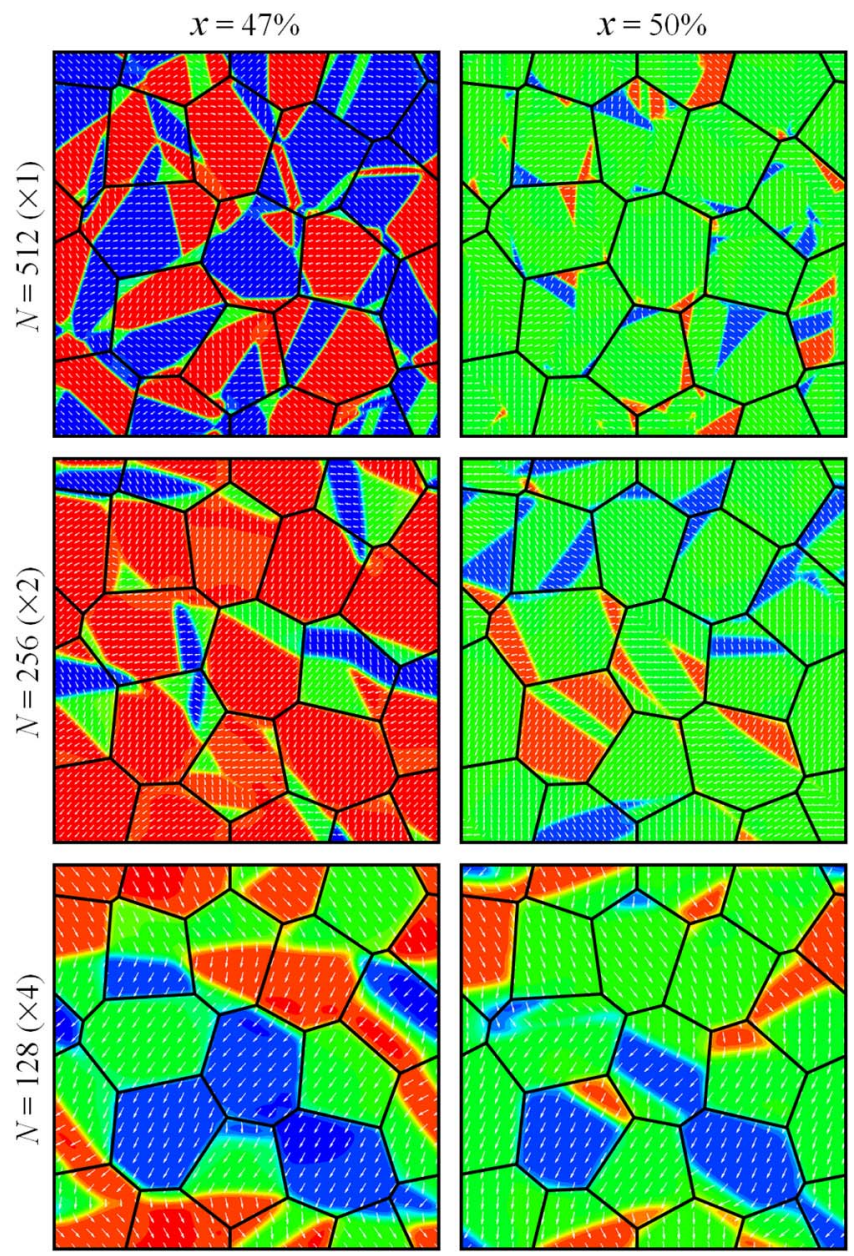

FIG. 2. (Color online) Computer simulation of grain size- and compositiondependent phase-coexisting domain microstructures around MPB in ferroelectric polycrystals. Same polycrystalline grain structures with different grain sizes and compositions are simulated. Smaller grains are enlarged four and two times, respectively, in visualization for clarity. Arrows represent the in-plane components of polarization vector, and the out-of-plane component is represented by color, where green domains are tetragonal phase, red and blue domains are rhombohedral phase. simulated by using different numbers of discrete computational grids $N \times N$ with $N=128,256$, and 512, respectively. A range of composition around $\mathrm{MPB}, 0.40 \leqslant x \leqslant 0.56$, is simulated. For better comparison with experimental results, statistical average is performed among ten phase volume fraction values that are individually simulated with different random initial conditions for same specific composition and grain size. This procedure is necessary because the simulation volume only represents a small portion of polycrystalline ceramic or powder samples used in diffraction experiments (a conventional technique for phase characterization), where different parts of the samples develop different domain microstructures and diffraction experiments measure the average phase volume fraction.

Figure 1 plots the volume fraction of tetragonal phase versus the composition $x$ for different grain sizes. It shows that the tetragonal phase fraction smoothly increases across the MPB from rhombohedral side to tetragonal side. Thus, tetragonal and rhombohedral phases coexist over a composition range around the MPB. The simulated data (open symbols) are widely scattered for near-MPB compositions and become narrowly distributed for compositions away from the MPB. Nevertheless, the average data (filled symbols) well follow the following function:

$$
f\left(x_{0}, \tau ; x\right)=\frac{\exp \left[\left(x-x_{0}\right) / \tau\right]}{1+\exp \left[\left(x-x_{0}\right) / \tau\right]},
$$

where the parameter $\tau$ measures the width of phase coexistence composition range. Most importantly, Fig. 1 reveals a grain size effect of phase coexistence around the MPB in ferroelectric polycrystals. The width of phase coexistence composition range increases with decreasing grain size, as quantified by the fitted values of $\tau$ in Table I. Further analysis by using asymmetric fitting function $g\left(x_{0}, \tau_{1}, \tau_{2} ; x\right)=[1-h(x$ $\left.\left.-x_{0}\right)\right] f\left(x_{0}, \tau_{1} ; x\right)+h\left(x-x_{0}\right) f\left(x_{0}, \tau_{2} ; x\right)$, where $h(x)$ is Heaviside step function, shows $\tau_{1}<\tau<\tau_{2}$, as listed in Table I. This behavior is consistent with the fact that the energy gap between metastable and stable phases increases slightly faster with composition deviation from MPB on rhombohedral side than on tetragonal side, according to the LGD polynomial ${ }^{24}$ used in our simulations. A larger energy gap increases the energy cost for the formation of metastable phase and decreases its volume fraction faster on the rhombohedral side 
TABLE I. Parameter values of the fitting functions $f\left(x_{0}, \tau ; x\right)$ and $g\left(x_{0}, \tau_{1}, \tau_{2} ; x\right)$.

\begin{tabular}{cccccc}
\hline \hline$N$ & $x_{0}$ & $\tau$ & $x_{0}$ & $\tau_{1}$ & $\tau_{2}$ \\
\hline 128 & 0.4891 & 0.0223 & 0.4878 & 0.0207 & 0.0242 \\
256 & 0.4867 & 0.0099 & 0.4863 & 0.0094 & 0.0103 \\
512 & 0.4878 & 0.0035 & 0.4876 & 0.0032 & 0.0038 \\
\hline \hline
\end{tabular}

of MPB. The underlying mechanisms are revealed in Fig. 2.

Figure 2 shows representative phase-coexisting domain microstructures in polycrystals from individual simulations for different compositions and grain sizes. As in the case of single crystals, ${ }^{11}$ due to the small bulk free energy gap between metastable and stable phases in the vicinity of MPBs, minor domains of metastable phase spontaneously coexist with and bridge major domains of stable phase and together form mosaic domain microstructures. The existence of minor phase as bridging domains provides extra configurational degrees of freedom for domain self-accommodation and reduces elastostatic, electrostatic, and polarization gradient (domain wall) energies of complex domain microstructures, which arise from the frustrations caused by both the crystal lattice misfit and polarization distribution among multiple polar domain variants. With compositions deviated from the $\mathrm{MPB}$, the bulk energy gap becomes large, thus, the energy cost of minor phase formation is high and the volume fraction of metastable phase decreases. This mechanism gives rise to the composition-dependent phase coexistence behavior, as described by Eq. (2). In polycrystals, the grain boundaries impose internal mechanical and electric boundary conditions, which affect the phase-coexisting domain microstructures in the grains. High internal stresses usually develop around grain boundaries and especially their junctions, where electric charges also accumulate. As a result, minor domains of metastable phase preferably form at grain boundaries and their junctions, as shown in Fig. 2. It also shows that the effects of grain boundaries are more significant for smaller grains. This mechanism explains the grain size-dependent phase coexistence behavior. Phases coexist over a wider composition range around MPB in polycrystals of smaller grains, where the larger internal stresses and charge accumulation tend to stabilize the bridging domains of minor phases.

The simulation results presented in Figs. 1 and 2 show that the domain microstructures and phase volume fractions vary widely from grain to grain. Thus, experimental observations based on imaging of local domain microstructures may not provide a reliable measurement of the volume fractions of coexisting phases in the whole polycrystalline sample. The conventionally used diffraction method is the appropriate technique to characterize phase coexistence and measure the average amount of each phase. The findings also suggest that MPB phase coexistence could be engineered through control of grain sizes, i.e., phase coexistence can be stabilized in fine-grained polycrystalline ceramics, while suppressed in coarse-grained materials.

In summary, computer modeling and simulation reveals a grain size- and composition-dependent behavior of phase coexistence around the MPBs in polycrystals of ferroelectric solid solutions. This phenomenon arises from the long-range electrostatic and elastostatic interactions in complex domain microstructures and the internal mechanical and electric boundary conditions associated with grain boundaries.

Support from NSF under Grant No. DMR-0705947 is acknowledged. The parallel computer simulations were performed on System X at Virginia Tech and Data Star at San Diego Supercomputer Center.

${ }^{1}$ B. Jaffe, W. R. Cook, and H. Jaffe, Piezoelectric Ceramics (Academic, London, 1971).

${ }^{2}$ J. Kuwata, K. Uchino, and S. Nomura, Ferroelectrics 37, 579 (1981); Jpn. J. Appl. Phys., Part 1 21, 1298 (1982).

${ }^{3}$ S. W. Choi, T. R. Shrout, S. J. Jang, and A. S. Bhalla, Ferroelectrics $\mathbf{1 0 0}$, 29 (1989); T. R. Shrout, Z. P. Chang, N. Kim, and S. Markgraf, Ferroelectr., Lett. Sect. 12, 63 (1990).

${ }^{4}$ S. E. Park and T. R. Shrout, J. Appl. Phys. 82, 1804 (1997).

${ }^{5}$ V. A. Isupov, Sov. Phys. Solid State 10, 989 (1968); Phys. Solid State 43, 2262 (2001); Ferroelectrics 266, 91 (2002).

${ }^{6}$ L. Benguigui, Solid State Commun. 11, 825 (1972); P. Ari-Gur and L. Benguigui, ibid. 15, 1077 (1974).

${ }^{7}$ K. Kakegawa, J. Mohri, T. Takahashi, H. Yamamura, and S. Shirasaki, Solid State Commun. 24, 769 (1977); K. Kakegawa, J. Mohri, S. Shirasaki, and K. Takahashi, J. Am. Ceram. Soc. 65, 515 (1982).

${ }^{8}$ W. Cao and L. E. Cross, Phys. Rev. B 47, 4825 (1993).

${ }^{9}$ A. M. Glazer, P. A. Thomas, K. Z. Baba-Kishi, G. K. H. Pang, and C. W. Tai, Phys. Rev. B 70, 184123 (2004).

${ }^{10}$ G. A. Rossetti, Jr., W. Zhang, and A. G. Khachaturyan, Appl. Phys. Lett. 88, 072912 (2006); G. A. Rossetti, Jr. and A. G. Khachaturyan, ibid. 91, 072909 (2007).

${ }^{11}$ W. F. Rao and Y. U. Wang, Appl. Phys. Lett. 90, 182906 (2007).

${ }^{12}$ W. F. Rao and Y. U. Wang, Appl. Phys. Lett. 91, 052901 (2007).

${ }^{13}$ For complete references to the intermediate MPB phases, see recent review, in B. Noheda and D. E. Cox, Phase Transitions 79, 5 (2006).

${ }^{14}$ Y. M. Jin, Y. U. Wang, A. G. Khachaturyan, J. F. Li, and D. Viehland, Phys. Rev. Lett. 91, 197601 (2003); J. Appl. Phys. 94, 3629 (2003).

${ }^{15}$ Y. U. Wang, Phys. Rev. B 73, 014113 (2006); 74, 104109 (2006); 76, 024108 (2007).

${ }^{16}$ H. Wang, J. Zhu, N. Lu, A. A. Bokov, Z. G. Ye, and X. W. Zhang, Appl. Phys. Lett. 89, 042908 (2006); H. Wang, J. Zhu, X. W. Zhang, Y. X. Tang, and H. S. Luo (unpublished).

${ }^{17}$ L. A. Schmitt, K. A. Schönau, R. Theissmann, H. Fuess, H. Kungl, and M. J. Hoffmann, J. Appl. Phys. 101, 074107 (2007); R. Theissmann, L. A. Schmitt, J. Kling, R. Schierholz, K. A. Schönau, H. Fuess, M. Knapp, H. Kungl, and M. J. Hoffmann, ibid. 102, 024111 (2007); K. A. Schönau, L. A. Schmitt, M. Knapp, H. Fuess, R. A. Eichel, H. Kungl, and M. J. Hoffmann, Phys. Rev. B 75, 184117 (2007); K. A. Schönau, M. Knapp, H. Kungl, M. J. Hoffmann, and H. Fuess, ibid. 76, 144112 (2007).

${ }^{18}$ Y. L. Li, S. Choudhury, Z. K. Liu, and L. Q. Chen, Appl. Phys. Lett. 83, 1608 (2003); Y. L. Li and L. Q. Chen, ibid. 88, 072905 (2006); S. Choudhury, Y. Li, and L. Q. Chen, J. Am. Ceram. Soc. 88, 1669 (2005).

${ }^{19}$ S. Semenovskaya and A. G. Khachaturyan, J. Appl. Phys. 83, 5125 (1998); Ferroelectrics 206-207, 157 (1998).

${ }^{20}$ Y. M. Jin, A. Artemev, and A. G. Khachaturyan, Acta Mater. 49, 2309 (2001); Y. U. Wang, Y. M. Jin, and A. G. Khachaturyan, ibid. 52, 1039 (2004).

${ }^{21}$ Y. M. Jin, Y. U. Wang, A. Kazaryan, Y. Wang, D. E. Laughlin, and A. G. Khachaturyan, J. Appl. Phys. 92, 6172 (2002).

${ }^{22}$ S. Choudhury, Y. L. Li, C. E. Krill III, and L. Q. Chen, Acta Mater. 53, 5313 (2005); 55, 1415 (2007).

${ }^{23}$ A. F. Devonshire, Philos. Mag. 40, 1040 (1949).

${ }^{24}$ A. Amin, M. J. Haun, B. Badger, H. McKinstry, and L. E. Cross, Ferroelectrics 65, 107 (1985). 